\title{
Inhibition of inflammatory responses elicited by urban fine dust particles in keratinocytes and macrophages by diphlorethohydroxy- carmalol isolated from a brown alga Ishige okamurae
}

\author{
I. P. Shanura Fernando, Hyun-Soo Kim, K. K. Asanka Sanjeewa, Jae-Young Oh, You-Jin \\ Jeon and Won Woo Lee*
}

Department of Marine Life Science, Jeju National University, Jeju 63243, Korea

\begin{abstract}
Fine dust (FD) particles have become a major contributor to air pollution causing detrimental effects on the respiratory system and skin. Although some studies have investigated the effects of FD on the respiratory system, their possible effects on the skin remain under-explored. We investigated the FD mediated inflammatory responses in keratinocytes, present in the outer layers of skin tissues and the transfer of inflammatory potential to macrophages. We further evaluated the anti-inflammatory effects of the polyphenolic derivative, diphlorethohydroxycarmalol (DPHC) isolated from Ishige okamurae against FD-induced inflammation. Size distribution of FD particles was analyzed by scanning electron microscopy. FD particles induced the production of cyclooxygenase-2, prostaglandin E2 ( $\mathrm{PGE}_{2}$ ), interleukin (IL)-1 3 , and IL-6 in HaCaT keratinocytes and the expression of nitric oxide (NO), inducible nitric oxide synthases (iNOS), PGE $_{2}$, tumor necrosis factor- $\alpha$ expression in RAW 264.7 macrophages. Further, we evaluated the inflammatory potential of the culture medium of inflammation-induced HaCaT cells in RAW 264.7 macrophages and observed a marked increase in the expression of NO, iNOS, $\mathrm{PGE}_{2}$, and proinflammatory cytokines. DPHC treatment markedly attenuated the inflammatory responses, indicating its effectiveness in suppressing a broad range of inflammatory responses. It also showed antiinflammatory potential in in-vivo experiments using FD-stimulated zebrafish embryos by decreasing NO and reactive oxygen species production, while eventing cell death caused by inflammation.
\end{abstract}

Key Words: diphlorethohydroxycarmalol; fine dust; HaCaT; inflammation; Ishige okamurae; RAW 264.7 macrophages; zebrafish

\section{INTRODUCTION}

Air pollution due to fine dust (FD) particles has recently become a subject of increasing concern in many parts of the world including eastern China, Korea, and Japan with detrimental effects on humans and other organisms. Rapid advances in industrialization, increased usage of petroleum-powered vehicles, heavy mining operations, coal-burning power plants and a host of other anthropogenic activities have greatly contributed to air pollution, they continue to adversely affect the environment and human health (Lee et al. 2015c). Air pollutants could include any toxic gas or airborne particle having an aerodynamic diameter less than $10 \mu \mathrm{m}$. These fine particles could be composed of both organic and inorganic compounds, and vary in size and composition based on
(1) \$ This is an Open Access article distributed under the terms of the Creative Commons Attribution Non-Commercial License (http://creativecommons.org/licenses/by-nc/3.0/) which permits unrestricted non-commercial use, distribution, and reproduction in any medium, provided the original work is properly cited.
Received July 18, 2017, Accepted August 14, 2017

* Corresponding Author

E-mail: 21cow@naver.com

Tel: +82-64-754-3475, Fax: +82-64-756-3493 
the source and environmental conditions. Vehicle smoke is a major contributor to air pollution, especially in cities. These emissions consist of volatile hydrocarbons, nitrogen oxides, airborne particles, and carbon monoxide (Pozzi et al. 2003). Smoke emitted by coal-burning power plants contains organic micropollutants such as polycyclic aromatic hydrocarbons, benzo[a]pyrene, benzene, toluene, and a host of other toxic gasses (Moreira dos Santos et al. 2004). These particles can cause a number of detrimental issues, including respiratory complications and are believed to elicit allergic reactions and inflammatory responses in RAW 264.7 macrophages (Nel et al. 1998, Monn and Becker 1999, Jalava et al. 2007). Mineral dust particles mainly originate from the soil due to natural processes such as weathering of soil and deflation (Schuetz 1989). They are primarily composed of oxides and carbonates of metals abundant in the soil. During spring, dust storms originating in Siberia and Mongolia carry the dust particles southeastward via strong northwesterlies, leading to the accumulation the dust particles mainly in low-pressure areas such as eastern China, Korea, and Japan (Lee et al. 2015c). In addition to natural sources, anthropogenic activities such as heavy mining operations, constructions, and a host of other activities that disturb the environmental balance, have indirectly contributed to the release of mineral dust into the atmosphere. Chronic exposure to dust stimulates the production of reactive oxygen species (ROS) and inflammation in macrophages (Vallyathan et al. 1988). Asbestos particles are responsible for lipid peroxidation in rat lung microsomes (Kandaswami et al. 1988) and many studies have been conducted concerning their effects on lungs (Doelman et al. 1990). However, the effect of these FD particles containing both inorganic and organic substances have not widely been studied for their effects on the skin. Some studies have described the association between airborne agents and skin diseases, such as contact dermatitis and skin irritations (Dooms-Goossens et al. 1986). Skin, the outermost barrier of the body is highly exposed to FD and other environmental factors. The open areas of the skin such as skin of the face, neck, arms, and lower legs are highly susceptible to diseases. In fact, the response of skin cells against oxidative stress, caused by these harmful agents, might trigger a series of detrimental effects that compromise the skin appearance. Hence, the identification of anti-inflammatory agents that could be applied on the skin, is of utmost necessity.

Marine algae-derived natural products have received a special attention in the past few years due to their potential biofunctional properties (Wijesinghe et al. 2014, Oh et al. 2016). Some of these algae are commonly consumed as functional foods owing to their nutraceutical properties (Eom et al. 2015, Lee et al. 2015a, 2015b, Sanjeewa et al. 2016b). They are a source of various functional ingredients, such as fucoidans, phycocoloides, fucoxanthins, and manny different polyphenolic compounds (Lee et al. 2015a, 2015b, Fernando et al. 2017b). Polyphenols from the natural products of brown algae are of particular interest due to their strong antioxidant effects and a variety of other bioactivities, including anti-inflammatory, antiallergic, anti-diabetic, and antibacterial activities (Fernando et al. 2016a). All polyphenols have a basic phenolic ring structure but yield a broad range of derivatives. Diphlorethohydroxycarmalol (DPHC) belongs to the class of-phlorotannins, which represent compounds made up of oligomers of phloroglucinol units. DPHC has been isolated from the marine brown alga Ishige okamurae and extensively explored for its functional properties, including antioxidant and radical scavenging properties, inhibition of $\alpha$-glucosidase and $\alpha$-amylase activity, inhibition of melanogenesis, UV and radioprotective effects, and anticancer and anti-inflammatory effects (Heo et al. 2008, 2009, 2010, Han et al. 2014).

The focus of the current study was to investigate the effect of FD particles on keratinocytes, the cells lining the outer layers of skin, and underlying macrophages, using a systematic approach that allows us to identify their effects on both cell lines separately. Further, we evaluated the anti-inflammatory potential of DPHC isolated from $I$. okamurae against the inflammatory responses induced by these FD particles.

\section{MATERIALS AND METHODS}

\section{Chemicals and reagents}

ERM certified FD reference material including ERMCZ100 (defined for organic constituents) and ERM-CZ120 (defined for inorganic constituents), 2', $7^{\prime}$-dichlorodihydrofluorescein diacetate (DCFH2-DA), acridine orange, 4-amino-5-methylamino-2', $7^{\prime}$-difluorofluorescein diacetate (DAF-FM DA), and 3-(4,5-dimethylthiazol-2-yl)-2,5diphenyltetrazolium bromide (MTT) were purchased from Sigma-Aldrich (St. Louis, MO, USA). All analytical grade organic solvents including, hexane, chloroform, ethyl acetate, and methanol used during the extraction and purification were purchased from Sigma-Aldrich. RAW 264.7 macrophages and HACAT cells were purchased from Korean Cell Line Bank (KCLB, Seoul, Korea). 
<smiles>Oc1cc(O)cc(Oc2c(O)cc3c(c2O)Oc2cc(Oc4c(O)cc(O)cc4O)c(O)c(O)c2O3)c1</smiles>

Fig. 1. Molecular structure of diphlorethohydroxycarmalol.

Dulbecco's modified Eagle's medium (DMEM), fetal bovine serum (FBS), F12 media, and antibiotics (penicillin and streptomycin) were purchased from GIBCO Inc. (Grand Island, NY, USA). Antibodies were purchased from Santa Cruz Biotechnology (Santa Cruz, CA, USA), and cytokine kits were purchased from eBioscience (San Diego, CA, USA), R\&D Systems (Minneapolis, MN, USA), BD Opteia (San Diego, CA, USA), and Invitrogen (Carlsbad, CA, USA).

\section{Purification and isolation of DPHC from Ishige okamurae}

The isolation procedure for DPHC (Fig. 1) is described by (Kim et al. 2016). Briefly, the dry algae material was extracted with $70 \%$ ethanol, and the crude was suspended in water and sequentially partitioned between hexane, chloroform, and ethyl acetate. The ethyl acetate fraction was resolved and further separated by pre-optimized preparative centrifugal partition chromatography to obtain DPHC. The selected fractions were analyzed and confirmed by high-performance liquid chromatography (HPLC) LC-DAD-ESI/MS for purity and structure. DPHC was obtained as a yellowish-brown semi-solid. Based on HPLC LC-DAD-ESI/MS m/z value $\left(\mathrm{M}^{+}\right)$was 512.0589 for $\mathrm{C}_{24} \mathrm{H}_{16} \mathrm{O}_{13}$.

\section{Estimation of FD particle size by scanning elec- tron microscopy}

The FD specimens were mounted on double-sided carbon tape and sputter-coated with platinum in a Q150R rotary-pumped sputter coater (Quorum Technologies, Lewes, UK). The surface morphology of ERM-CZ100 and ERM-CZ120 FD particles was observed by a JSM-6700F field-emission scanning electron microscope (JEOL, Tokyo, Japan) operated at $10.0 \mathrm{kV}$.

\section{Maintenance of cell lines}

HaCaT (human keratinocyte), and RAW 264.7 macrophages were maintained in DMEM media supplemented with $10 \% \mathrm{FBS}$, and $1 \%$ antibiotics under a humidified atmosphere supplemented with $5 \% \mathrm{CO}_{2}$ at $37^{\circ} \mathrm{C}$. Cells were periodically subcultured, and the cells in log phase were used for the experiments.

\section{Evaluation of NO production in FD-induced RAW 264.7 macrophages}

FD was suspended in DMEM medium and homogenized by continuous sonication for $10 \mathrm{~min}$ achieving a final concentration of $2 \mathrm{mg} \mathrm{mL}^{-1}$. HaCaT keratinocytes and RAW 264.7 macrophages were seeded in 24-well plates with a $1 \times 10^{5}$ cells $\mathrm{mL}^{-1}$ concentration. After $24 \mathrm{~h}$ incubation, different concentrations of DPHC was treated into the wells. After $1 \mathrm{~h}$, the cells were stimulated by treating with $100 \mu \mathrm{g} \mathrm{mL} \mathrm{m}^{-1}$ of FD particles (both ERM-CZ100 and ERM-CZ120 FD separately). The concentration of FD for cell experiments were established as $100 \mu \mathrm{g} \mathrm{mL}^{-1}$ based on preliminary studies. In a separate setup, FD-induced HaCaT keratinocyte culture plates were treated with selected concentrations of DPHC and incubated for three hours. After, the culture media was replaced and further incubated for another $24 \mathrm{~h}$. Half of the HaCaT cell supernatants $(250 \mu \mathrm{L})$ were transferred to 24 -well culture plates containing pre-seeded RAW 264.7 macrophages and incubated for $24 \mathrm{~h}$. NO production and cell viability were measured by Gris and MTT assays as described by (Wijesinghe et al. 2014). The systematic experimental setup for the aforementioned cell culture experiments is depicted in Fig. 2.

\section{Western blot analysis}

Western blot analysis was carried out to determine the expression levels of pro and anti-inflammatory cytokines according to the method described by Sanjeewa et al. (2016a). Cytosolic proteins were extracted from the cells using lysis buffer. Proteins were standardized to $50 \mu \mathrm{g}$ for the electrophoresis using a BSA protein assay kit (Thermo Scientific, Waltham, MA, USA). The electrophoresis was carried out on $12 \%$ sodium dodecyl sulfate-polyacrylamide gels. The resolved bands were blotted onto nitrocellulose membranes and blocked with 5\% skim milk in TBST. Membranes were soaked in primary antibodies and kept under gentle shaking at $4^{\circ} \mathrm{C}$ for eight hours. Membranes were washed with TBST and gently shaken 


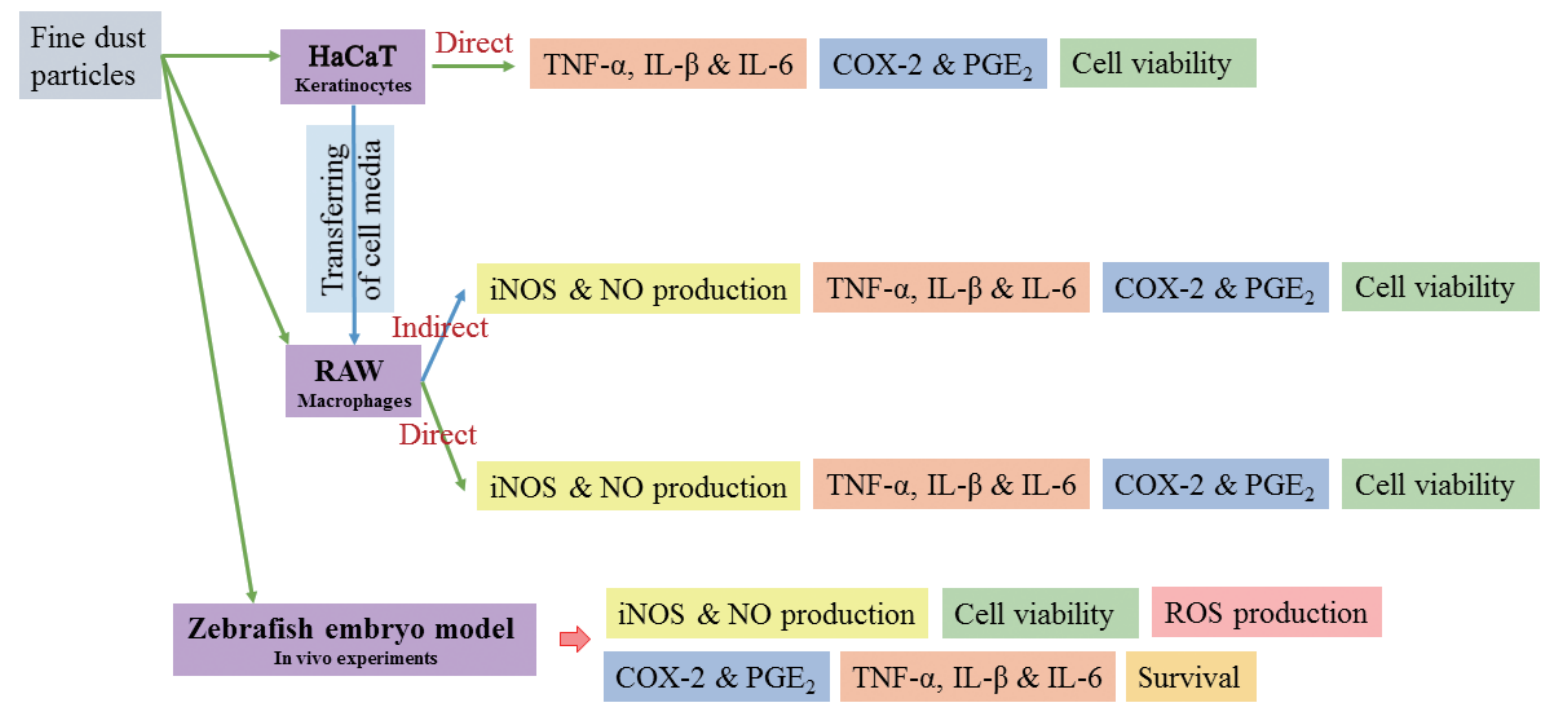

Fig. 2. Systematic experimental approach for evaluating anti-inflammatory effects using cell culture experiments. TNF-a, tumor necrosis factor $a$; IL, interleukin; COX-2, cyclooxygenase-2; $\mathrm{PGE}_{2}$, prostaglandin E2; iNOS, inducible nitric oxide synthases; ROS, reactive oxygen species.

for $3 \mathrm{~h}$ with the secondary antibodies at room temperature. After washing the membranes with TBST, the signals were developed by adding chemiluminescent substrate (Cyanagen Srl, Bologna, Italy). Images were obtained by a FUSION SOLO Vilber Lourmat system (Paris, France). The band intensities were quantified using Image J program.

\section{Analysis of cytokine production}

Cell culture experiments were carried out as described previously. Culture media was retrieved from each separate well after each test to analyze the expression of prostaglandin $\mathrm{E} 2\left(\mathrm{PGE}_{2}\right)$, tumor necrosis factor $\alpha$ (TNF- $\left.\alpha\right)$, interleukin (IL)-1 $\beta$, and IL-6. Experiments were done using enzyme immunoassay kits following the manufacturer's instructions.

\section{Zebrafish embryo experiments}

Zebrafish embryos were obtained by inducing natural spawning with the onset of light. Experiments were carried out to evaluate the FD-induced ROS, NO production, and cell death in zebrafish embryo model as described by (Kim et al. 2014). Briefly, the embryos were transferred to 12-well plates (15 embryos well ${ }^{-1}$ ) and treated with samples followed by FD-stimulation. After three days, the hatched larvae were separately stained using different fluorescence probe dyes including DAF-FM DA, 2,7-dichlorofluorescein diacetate (DCF-DA) and acridine orange respectively to identify NO, ROS levels, and cell death. The images were taken using a fluorescent microscope, equipped with a Moticam digital camera (British Columbia, Canada). Fluorescence intensities were quantified using Image J program.

\section{Statistical analysis}

Data values are given as mean \pm SD based on triplicate determinations. Statistical analysis of the data was performed using IBM SPSS Statistics ver. 20 software (IBM Corp., Armonk, NY, USA) for comparing the data using one-way ANOVA by Duncan's multiple range test. p-values less than $0.05(\mathrm{p}<0.05)$ were considered as significant.

\section{RESULTS}

\section{Size distribution and chemical properties of FD particles}

Fig. 3 shows the scanning electron microscope images of the ERM-CZ100 and ERM-CZ120 FD particles. The ERM-CZ120 and ERM-CZ100 FD particles had maximum diameters in the range of 11-16 $\mu \mathrm{m}$ and $15-20 \mu \mathrm{m}$, respectively. However, a large proportion of the particles had diameters $<3 \mu \mathrm{m}$. The particle morphologies were 

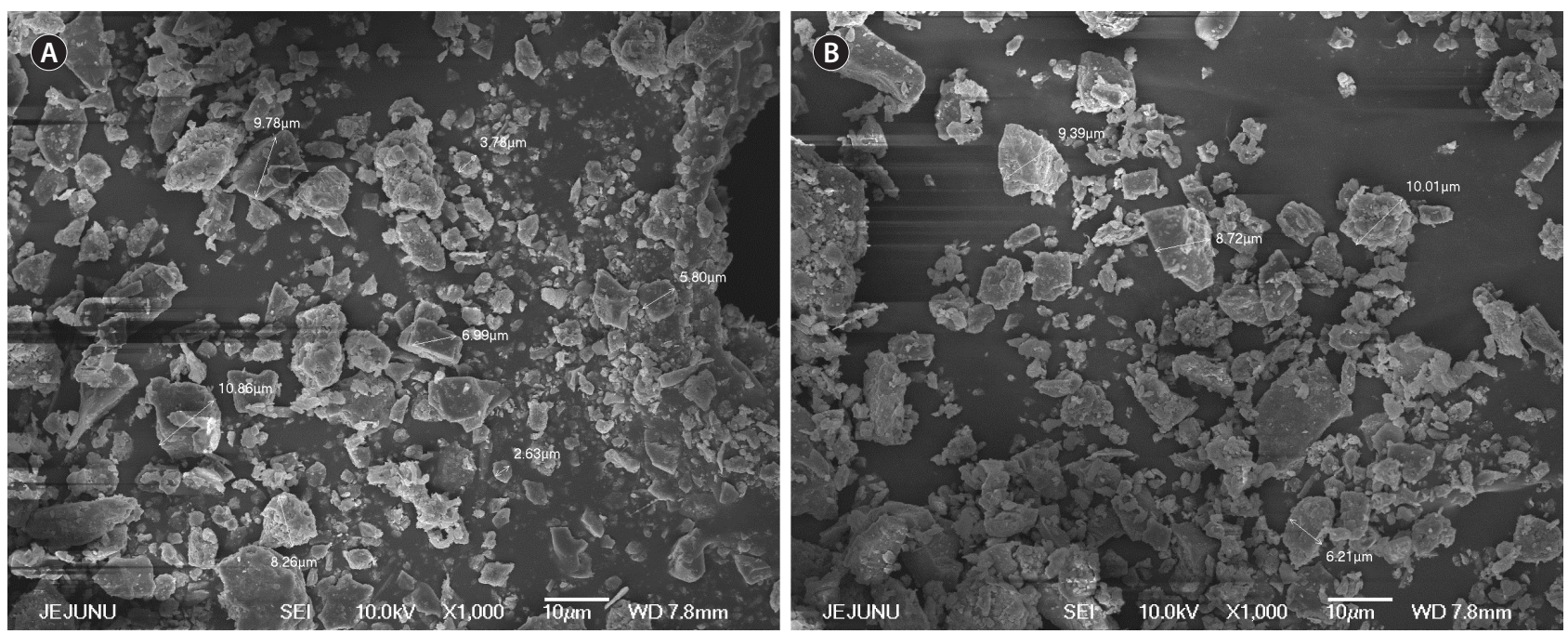

Fig. 3. Scanning electron microscope images of ERM-CZ120 (A) and ERM-CZ100 (B) fine dust (FD) particles. Scale bars represent: $A \& B, 10 \mu m$.

inconsistent throughout the sample, with irregularly shaped crystalline, semi-crystalline and granulated particles with rough surfaces; ERM-CZ120 FD had a higher number of crystalline and semi-crystalline, particles than ERM-CZ100. FD particles used in this study were produced and standardized by the Institute for Reference Materials and Measurements of the Joint Research Center of European Commission. The partials have been collected from the tunnel walls of a road tunnel in Wislostrada, Poland. ERM-CZ100 was certified to contain seven polycyclic aromatic hydrocarbons: benzo[a]anthracene, benzo[k]fluoranthene, benzo[b]fluoranthene, benzo[j] fluoranthene, benzo[a]pyrene, indeno[123-c,d]pyrene, and dibenzo[a,h]anthracene. ERM-CZ120 was certified to contain four elements: $\mathrm{As}, \mathrm{Cd}, \mathrm{Ni}$, and $\mathrm{Pb}$.

\section{FD-induced inflammation in $\mathrm{HaCaT}$ keratino- cytes and anti-inflammatory effects of DPHC}

In the current study, the levels of inflammatory regulators, including $\mathrm{PGE}_{2}$ and thereby cyclooxygenase-2 (COX-2) and the pro-inflammatory cytokines, IL-1 $\beta$ and IL-6 were significantly up-regulated following lipopolysaccharide (LPS; $1 \mu \mathrm{g} \mathrm{mL}^{-1}$ ) and FD treatment (100 $\left.\mu \mathrm{g} \mathrm{mL}^{-1}\right)$. LPS, the most commonly used inflammatory stimulator, was utilized as a reference to determine the inflammatory effects of FD. The phenolic derivative DPHC, dose-dependently downregulated the expression of $\mathrm{PGE}_{2}$, COX-2, IL-1 $\beta$, and IL-6 (Fig. 4) at concentrations of $6.25,12.50$, and $25.00 \mu \mathrm{g} \mathrm{mL}{ }^{-1}$. These concentrations were based on the cytotoxicity data for RAW 264.7 macrophages, as described in the upcoming section. However, both FD and LPS stimulation only negligibly altered the production (shallow expression level) of TNF- $\alpha$ (25.58 \pm $24.73 \mathrm{pg} \mathrm{mL}^{-1}$ ) in HaCaT keratinocytes following an incubation period of $24 \mathrm{~h}$, indicating that HaCaT keratinocytes do not produce TNF- $\alpha$ upon LPS or FD stimulation. A similar study reported that LPS stimulation (10-1,000 ng $\mathrm{mL}^{-1}$ ) does not significantly alter TNF- $\alpha$ production in HaCaT cells (Zbytek et al. 2002).

\section{FD-induced inflammation in RAW 264.7 macro- phages and anti-inflammatory effects of DPHC}

As shown in Fig. 5, direct stimulation by both FD particles and LPS, increased NO production in RAW 264.7 macrophages; however, the increase was higher after stimulation by LPS than that after stimulation by FD particles. DPHC treatment, efficiently and dose-dependently, suppressed NO production in FD-induced RAW 264.7 macrophages. Both LPS and FD exhibited toxicity effects in RAW 264.7 macrophage as indicated by the decreased viabilities. The initial increase in RAW 264.7 macrophage viability at DPHC concentrations of 1.125, 6.25, 12.50, and $25.00 \mu \mathrm{g} \mathrm{mL}^{-1}$ shows that DPHC has a protective effect against the toxicity induced by FD. However, DPHC (at a concentration of $50.0 \mu \mathrm{g} \mathrm{mL}^{-1}$ ) rapidly decreased RAW 264.7 macrophages viability. Hence, DPHC concen- 


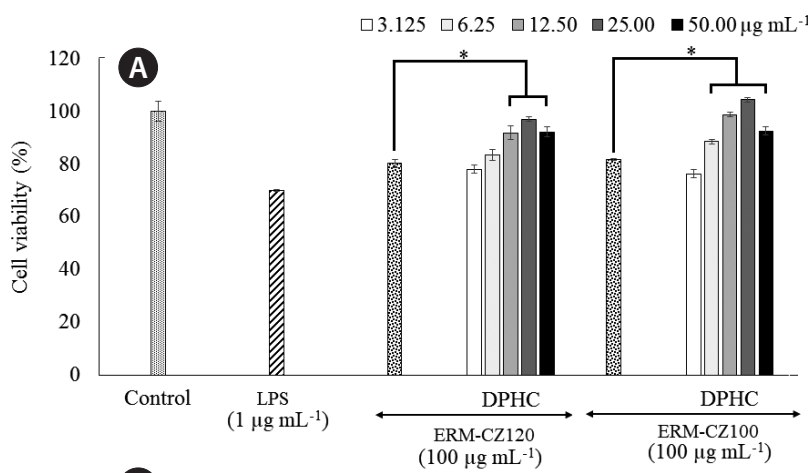

B
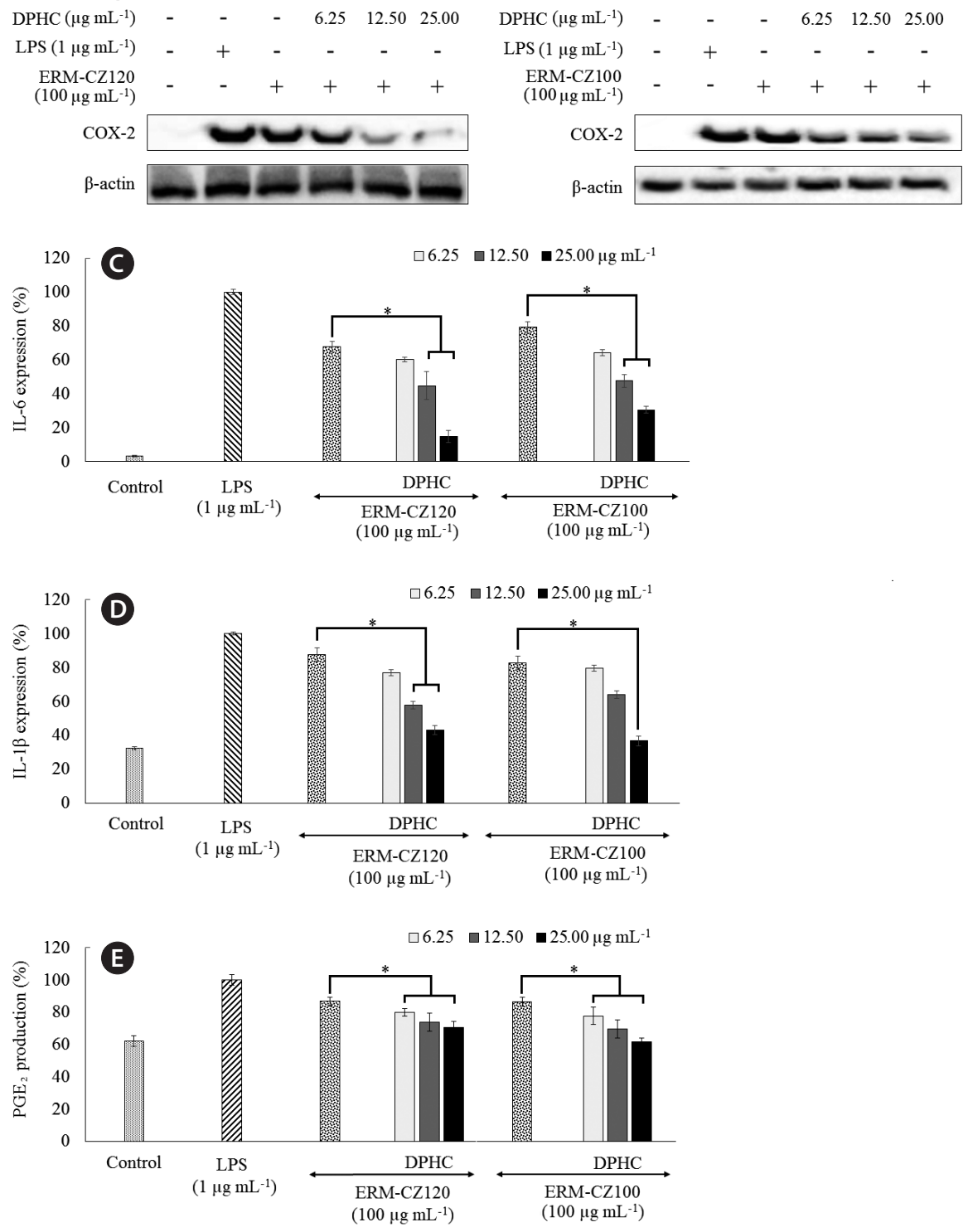

Fig. 4. Inflammatory stimulation of HaCaT keratinocytes by fine dust (FD) and anti-inflammatory effects of diphlorethohydroxycarmalol (DPHC): cytotoxicity of FD particles (A), analysis of cyclooxygenase-2 (COX-2) level (B), and inflammatory mediators including interleukin (IL)-6 (C), IL-1 $\beta$ (D), and prostaglandin E2 $\left(\mathrm{PGE}_{2}\right)(\mathrm{E})$. The cells were pre-seeded in culture plates $\left(1 \times 10^{5}\right.$ cells $\left.\mathrm{mL}^{-1}\right)$, incubated for $24 \mathrm{~h}$, and treated with different concentrations of DPHC. After $1 \mathrm{~h}$, the wells were treated with either ERM-CZ100 or ERM-CZ120 FD particles $\left(100 \mu \mathrm{gL}^{-1}\right)$ and $24 \mathrm{~h}$ later, the plates were used for the analysis of inflammatory mediators and cytotoxicity. Experiments were carried out in triplicate, and the results are represented as means \pm SD. Values are significantly different from the positive control (FD treated group) at ${ }^{*} p<0.05$. LPS, lipopolysaccharide. 

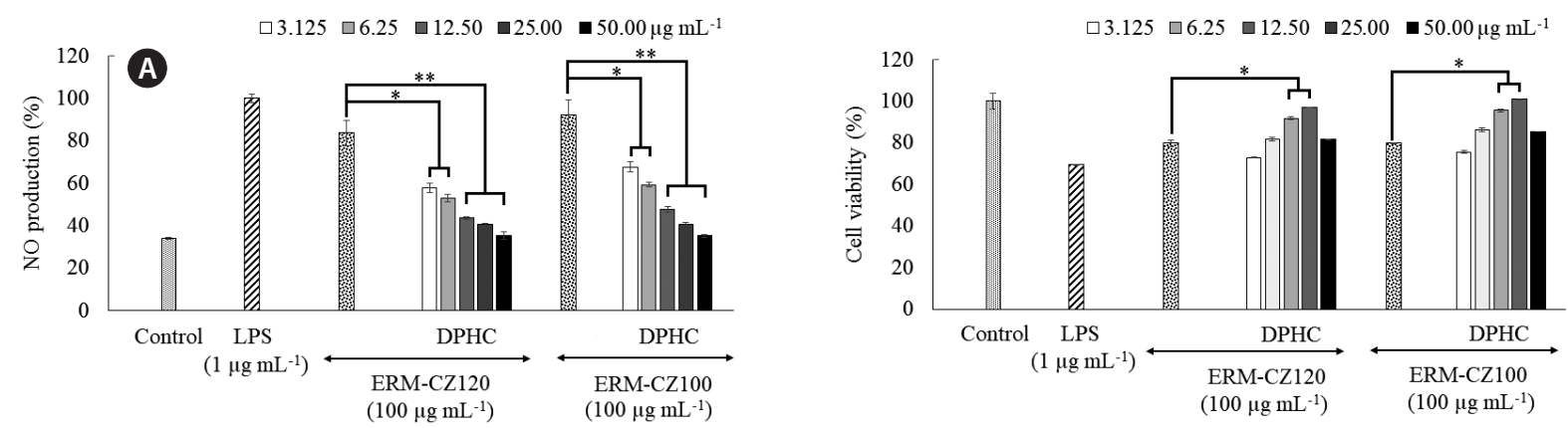

B
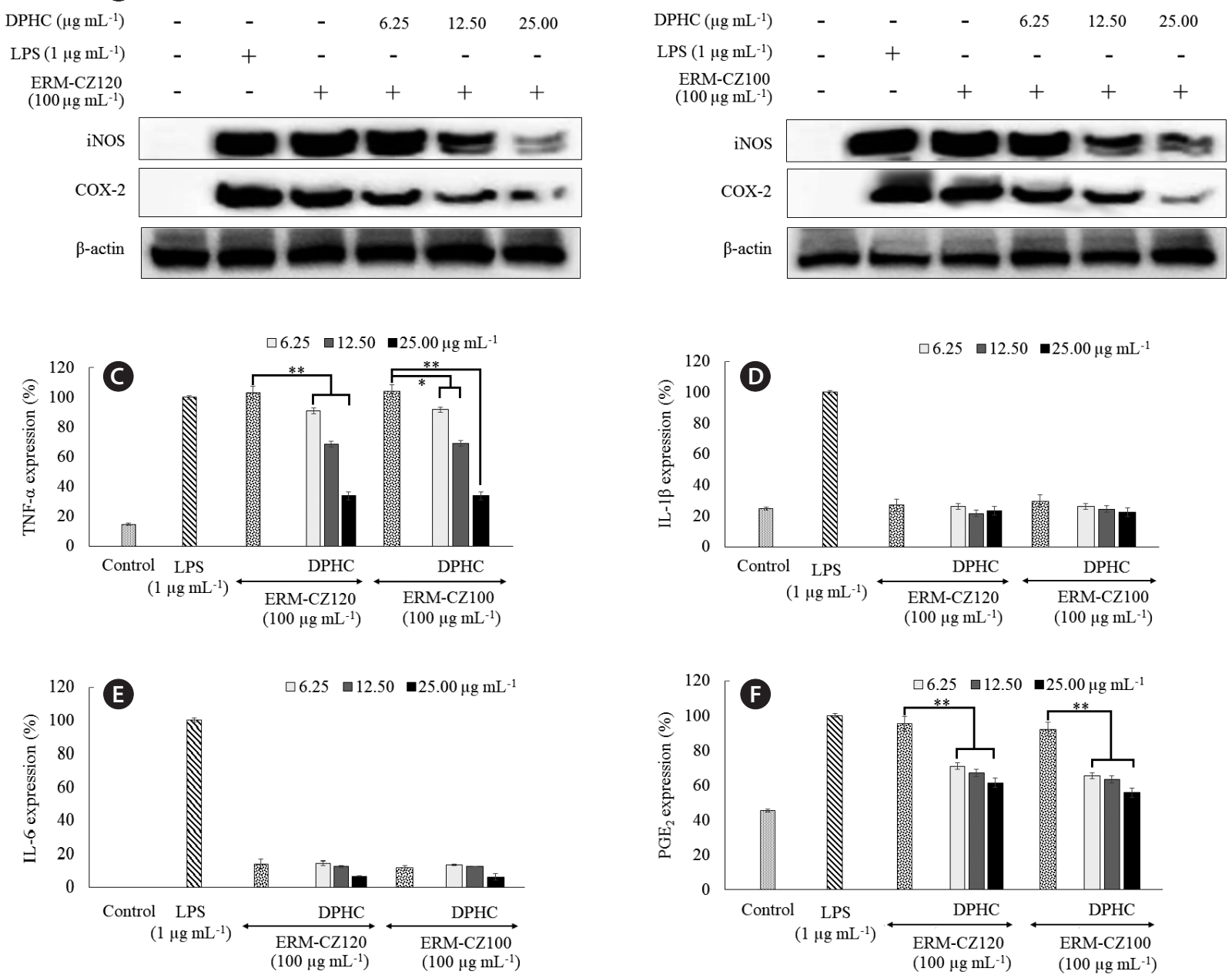

Fig. 5. Inflammatory stimulation of RAW 264.7 macrophages by fine dust (FD) and anti-inflammatory effects of diphlorethohydroxycarmalol (DPHC): NO production and cytotoxicity of FD particles (A), and analysis of inducible nitric oxide synthases (iNOS) and cyclooxygenase-2 (COX-2) (B), and inflammatory mediators, including tumor necrosis factor a (TNF-a) (C), interleukin (IL)-1 $\beta$ (D), IL-6 (E), and prostaglandin E2 (PGE 2 ) (F). The cells were pre-seeded in culture plates $\left(1 \times 10^{5}\right.$ cells mL $\left.{ }^{-1}\right)$, incubated for $24 \mathrm{~h}$, and treated with different concentrations of DPHC for $1 \mathrm{~h}$. The wells were then treated with either ERM-CZ100 or ERM-CZ120 FD particles $\left(100 \mu \mathrm{g} \mathrm{mL}^{-1}\right)$ and $24 \mathrm{~h}$ later, the plates were used for the analysis of inflammatory mediators and cytotoxicity. Experiments were carried out in triplicate, and the results are represented as means \pm SD. Values are significantly different from the positive control (FD treated group) at ${ }^{*} p<0.05$ and ${ }^{* *} p<0.001$. LPS, lipopolysaccharide. 

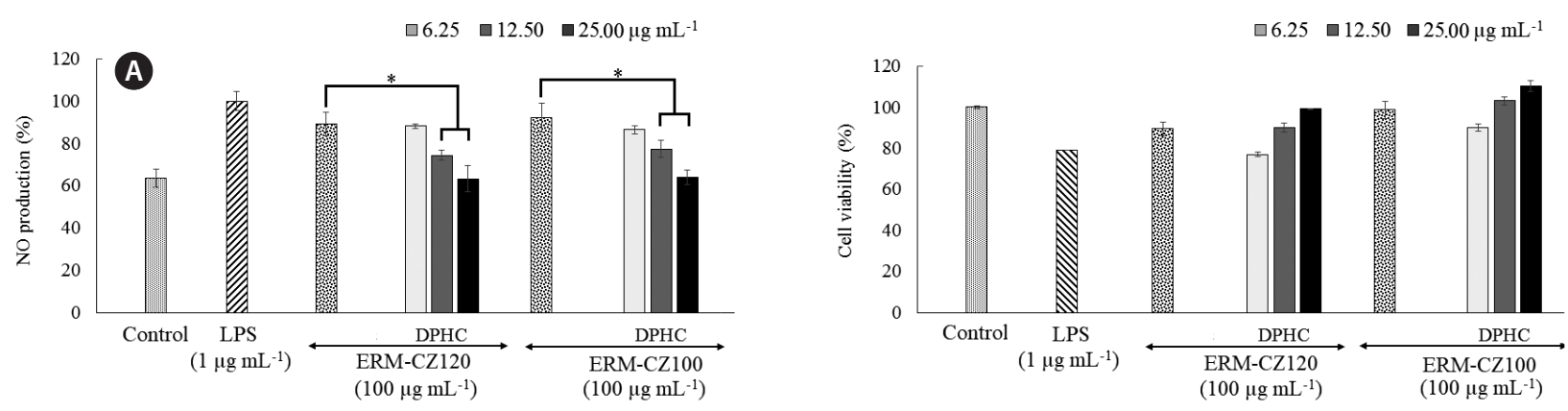

B
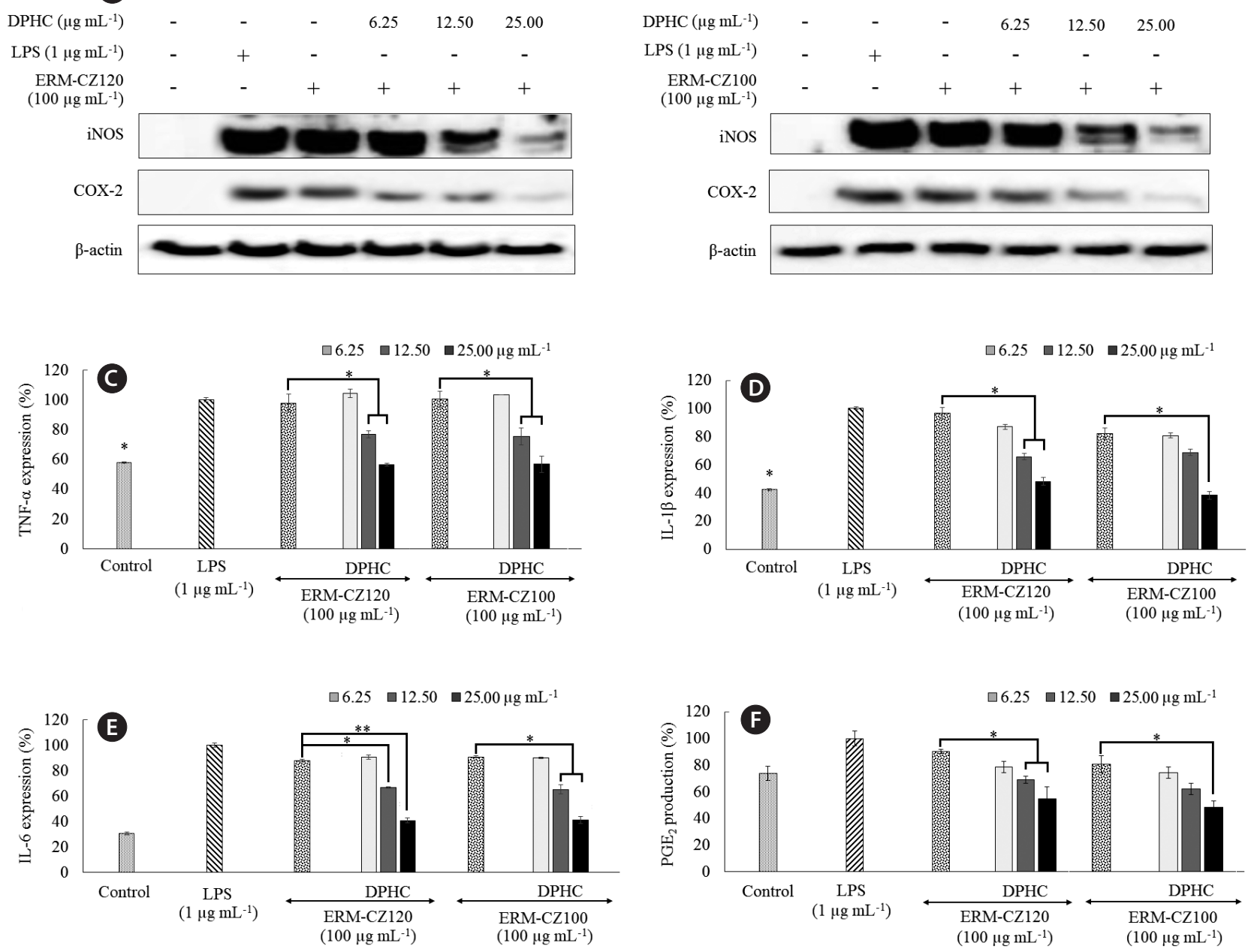

Fig. 6. Inflammatory stimulation of the RAW 264.7 macrophages by the culture medium of fine dust (FD)-induced HaCaT cells and the antiinflammatory effects of diphlorethohydroxycarmalol (DPHC): NO production and cytotoxicity (A), and analysis of inducible nitric oxide synthases (iNOS) and cyclooxygenase-2 (COX-2) levels (B) and inflammatory mediators, including tumor necrosis factor a (TNF-a) (C), interleukin (IL)-1 $\beta$ (D), IL-6 (E), and prostaglandin E2 (PGE $)(F)$. The RAW 264.7 macrophages were pre-seeded in culture plates $\left(1 \times 10^{5}\right.$ cells $\left.\mathrm{mL}^{-1}\right)$, incubated for $24 \mathrm{~h}$, and treated with different concentrations of DPHC. After $1 \mathrm{~h}$, the cells were treated with either ERM-CZ100 or ERM-CZ120 FD particles $\left(100 \mu \mathrm{g} \mathrm{mL} \mathrm{L}^{-1}\right)$ and $24 \mathrm{~h}$ later, the culture media were transferred to pre-seeded RAW 264.7 macrophages culture well plates. The evaluations were made after a $24 \mathrm{~h}$ period. Experiments were carried out in triplicate, and the results are represented as means $\pm \mathrm{SD}$. Values are significantly different from the positive control (FD treated group) at ${ }^{*} \mathrm{p}<0.05$ and ${ }^{* *} \mathrm{p}<0.001$. LPS, lipopolysaccharide. 

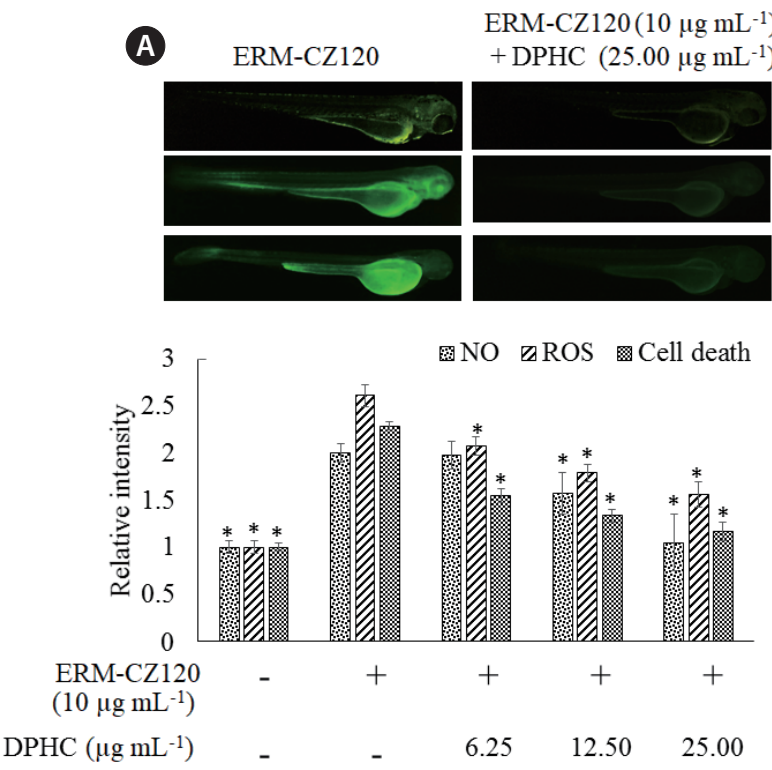
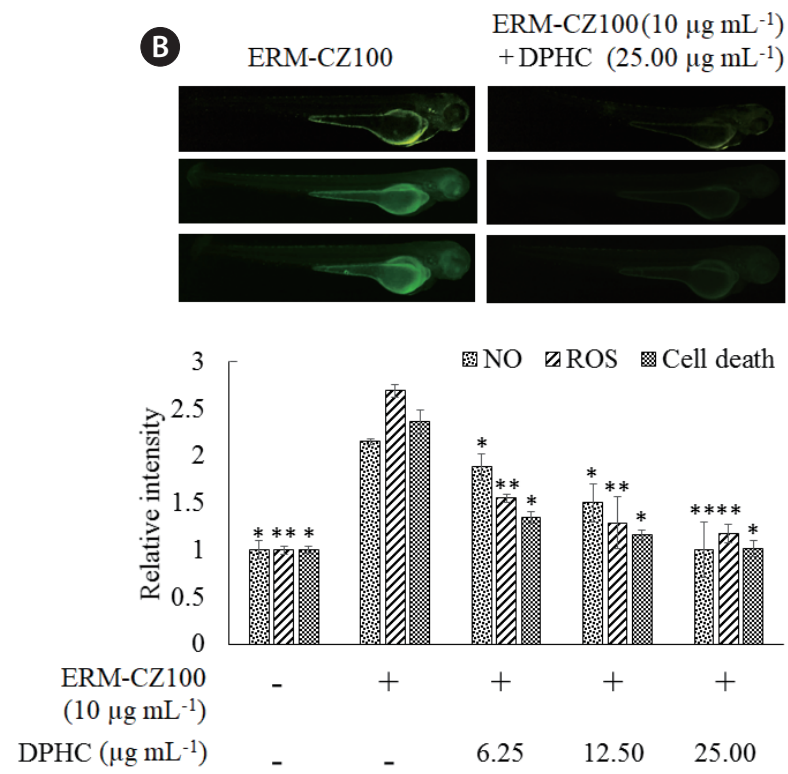

Fig. 7. Inflammatory stimulation of zebrafish larvae by fine dust (FD) and anti-inflammatory effects of diphlorethohydroxycarmalol (DPHC): anti-inflammatory properties were evaluated by measuring NO and reactive oxygen species (ROS) production, and cell death in the zebrafish embryo model. Effects of ERM-CZ120 FD (A) and ERM-CZ100 FD (B) particles and anti-inflammatory effects of DPHC. Experiments were carried out in triplicate, and the results are represented as means \pm SD. Values are significantly different from the positive control (FD treated group) at * $p<0.05$ and ${ }^{* *} p<0.001$.

trations of $6.25,12.50$, and $25.00 \mu \mathrm{g} \mathrm{mL} \mathrm{L}^{-1}$ were selected for further studies. Western blot analysis showed that FD stimulation rapidly increased inducible nitric oxide synthases (iNOS) and COX-2 protein levels, whereas treatment with DPHC dose-dependently downregulated their levels. iNOS and COX-2 are two of the major inflammatory regulators that indirectly control the production of $\mathrm{NO}$ and $\mathrm{PGE}_{2}$. Consistent with these observations, we observed the levels of $\mathrm{NO}$ and $\mathrm{PGE}_{2}$ shown in Fig. 5. Moreover, FD stimulation notably upregulated the expression of TNF- $\alpha$ to a level comparable with LPS stimulation (Fig. $5 C)$. In fact, the protein level of IL- $1 \beta$ upon FD stimulation did not alter significantly from its basal value. However, FD stimulation caused a non-significant increase in the IL-6 levels compared to the control value. DPHC treatment dose-dependently decreased the IL-6 levels. All of these observations are in agreement with those reported by Jalava et al. (2007) except that FD stimulation increased IL-6 secretion in RAW 264.7 macrophages to a lesser extent than that reported. Interestingly, both ERM-CZ100 and ERM-CZ120 FD particles yielded similar results for cell culture experiments. This could be related to their similar origin whereas both particles might contain similar types of constituents apart from their defined (standardized) parameters given based on different analytical techniques.

\section{Ability of the culture medium of inflammation induced $\mathrm{HaCaT}$ cells to induce inflammatory re- sponses in RAW 264.7 macrophages and the role of DPHC as an anti-inflammatory agent}

To study the systematic relationship between keratinocytes and macrophages in transferring the inflammatory potential, we initially induced inflammation in keratinocytes by exposing them to FD for one hour. The cells were then co-treated with different concentrations of DPHC and further incubated for $3 \mathrm{~h}$ after which the culture medium was replaced. After $24 \mathrm{~h}$ of incubation, the media were transferred to RAW 264.7 macrophages. Cell viability, NO production, and levels of iNOS, COX-2, IL-6, IL-1 $\beta$, and TNF- $\alpha$ were evaluated. As shown in Fig. 6 , the culture media from the FD stimulated keratinocytes induced a higher NO production in RAW 264.7 macrophages to a level similar to that observed in LPS-stimulated HaCaT cells. Indirect stimulation of RAW 264.7 macrophages by FD, induced NO production to a greater extent (Fig. 6) than direct stimulation by FD did (Fig. 5). Interestingly, DPHC treatment effectively suppressed the inflammatory responses in a dose-dependent manner while increasing cell viability. Accordingly, a reduction in iNOS and COX2 levels was observed with increasing concentrations of DPHC (Fig. 6). Moreover, the levels of pro-inflammatory mediators were reduced by DPHC. Accordingly, DPHC 
dose-dependently reduced $\mathrm{PGE}_{2}$ to a level lower than that observed in the respective control groups indicating better anti-inflammatory potential.

According to a comprehensive study by Akdis et al. (2016), keratinocytes produce IL- $1 \alpha$, IL-1 $\beta$, IL-6, IL-7, IL8, IL-15, IL-18, IL-19, and IL-20 among which IL-18 has been proven to induce inflammatory responses in macrophages. According to Li and Verma (2002), pro-inflammatory cytokines including TNF and IL-1 could activate nuclear factor $\mathrm{\kappa B}$ signaling pathway initiating both innate and adaptive immune responses. Hence, IL-1 $\beta$ produced in HaCaT keratinocytes might have contributed for inducing inflammatory responses in RAW 264.7 macrophages.

\section{An in vivo zebrafish embryo model for study- ing the effects of FD-induced inflammation and anti-inflammatory effects of DPHC}

As shown in Fig. 7, stimulation with both types of FD rapidly increased ROS and NO production, and cell death in zebrafish larvae, thereby reducing the RAW cell viability. However, DPHC treatment markedly attenuated ROS and NO levels in the larvae and protected the cells against FD-induced cell death. These observations suggest that the zebrafish embryo model is effective for evaluating the inflammatory responses of FD particles. Moreover, DPHC dose-dependently exhibited a desirable anti-inflammatory activity against the FD-induced inflammatory responses in the zebrafish embryo model.

\section{DISCUSSION}

FD particles have become a major cause of air pollution in several parts of the world, mainly eastern China, Korea, and Japan due to the marked upsurge in industrialization, coal-burning power plants, vehicular traffic, heavy mining operations, sand storms, and other anthropogenic and natural events (Lee et al. 2015c). Although FD has become a major issue in the Southeast Asian region, only a few studies describe their size or chemical composition. One study describes the investigation of air pollutants in the upper atmosphere and nearby the background station in Taean, Korea. Accordingly, the air has been found to contain partials composed of $\mathrm{SO}_{4}{ }^{2-}, \mathrm{NO}_{3}$, $\mathrm{NH}_{4}{ }^{+}, \mathrm{Cl}, \mathrm{Na}^{+}, \mathrm{K}^{+}$, and $\mathrm{Mg}^{2+}$ ions (Kim et al. 2001). Another study describes the evaluation of FD of $<10 \mu \mathrm{m}$ collected in Seoul, South Korea during an Asian dust incident (Choi et al. 2001). The composition of the dust has been recorded as: $\mathrm{NO}_{3}{ }^{-}, \mathrm{SO}_{4}{ }^{2-}, \mathrm{Na}^{+}, \mathrm{K}^{+}, \mathrm{NH}_{4}{ }^{+}, \mathrm{Mg}^{2+}, \mathrm{Ca}^{2+}, \mathrm{Na}, \mathrm{Al}$, $\mathrm{Mg}$, Ca, Fe, Mn, Cr, Cu, Co, Zn, Pb, and Cd. An interesting study done in China describe the evaluation of chemical composition and anti-inflammatory effects of $\leq 2.5 \mu \mathrm{m}$ urban particulate matter collected from Shenyang city in China on RAW 264.7 macrophages (Bekki et al. 2016). The dust particles have increased the IL- $1 \beta$ and COX-2 expression levels in macrophage cells indicating strong inflammatory responses. The evidence support that both chemical composition and size of FD particles affects the inflammation inducing effects. According to Schwartz et al. (1996) the smaller the particle size higher would be its detrimental impact. Developing methods for investigating FD particles have received an increased attention as they cause a number of detrimental effects. Although numerous studies have discussed the effects of FD on the respiratory system, the present study evaluated its inflammatory potential in the keratinocytes of the skin tissues and RAW 264.7 macrophages. The dust ERM-CZ100 and ERM-CZ120 used in this study were collected from road dust near a tunnel in Wislostrada, Poland. This material could be mainly originating from the vehicle smoke (combustion of petroleum fuel) and mineral particles in soil. The material used in the present study indicate a close resemblance to the compounds identified from the urban dust collected in China by Bekki et al. (2016).

The keratinocyte model is commonly used to evaluate the effects of irritants in dermatology because these cells are located in the outer layers of the skin and are the first to encounter foreign irritants. They play a vital role in maintaining the stratum corneum barrier, thereby protecting the inner layers of skin cells. Keratinocytes have the ability to produce a broad range of inflammatory mediators (Williams and Kupper 1996) that further induce the upregulation and secretion of secondary mediators, including chemokines, causing the recruitment of leukocytes to the damaged area of the skin. The role of keratinocytes as initiators of inflammation has been comprehensively reviewed by Barker et al. (1991). Macrophages play a major role in regulating inflammation via phagocytosis, antigen presentation, and via the production of various growth factors and cytokines. Activation of macrophages is an essential strategy to counteract these detrimental conditions. However unregulated or continuous activation of macrophages results in detrimental outcomes. Activation of macrophages is regulated by cytokines, such as interferon $\gamma$, TNF- $\alpha$, and granulocytemonocyte colony stimulating factor, or by certain extracellular matrix components, such as lipopolysaccharide, and certain chemicals (Fujiwara and Kobayashi 2005). 
The activated macrophages regulate the inflammatory responses by mediating, the levels of NO, iNOS, COX-2, $\mathrm{PGE}_{2}$, and the pro-inflammatory cytokines, including TNF- $\alpha$, IL-1 $\beta$, and IL-6 (Fernando et al. 2016b). Based on the results of the present study, FD elicited inflammatory responses in keratinocytes and transferred the inflammatory responses to RAW 264.7 macrophages possibly mediated by the proinflammatory cytokine IL-1 $\beta$. Several studies have described the inflammation-stimulating effects of components in FD particles. According to Nel et al. (1998), diesel exhaust particles that contain polycyclic aromatic hydrocarbons induce allergic inflammation via inducing IgE production. Treatment of polyphenolic derivative DPHC isolated from the marine algae I. okamurae markedly attenuated the inflammatory responses indicating their effectiveness in suppressing a broad range of inflammatory effects. This study identified the skin-inflammatory potential of FD and demonstrated the anti-inflammatory potential of DPHC aganist inflammatory responses elicited by FD.

Recently zebrafish models have gained popularity over other animal models for stimulating human diseases. Several studies have shown the suitability of the LPS-induced zebrafish embryo model for evaluating inflammatory effects (Fernando et al. 2017a). However, this is the first study to use a FD-induced zebrafish embryo model for evaluating inflammatory effects. During the initial trials, larvae were observed to die upon hatching, possibly due to the blocking of larval gills by FD particles. Daily changing of the zebrafish embryo media and mounting in new embryo media until hatching reduced the larval mortality. Further, FD was used at a concentration (10 $\mu \mathrm{g}$ $\mathrm{mL}^{-1}$ ) lower than that used in in vitro cell culture experiments. These in vivo experiments indicated the ability of FD for inducing inflammatory responses in zebrafish embryos. These responses, however, could effectively counter with DPHC treatment at a concentration of $25 \mu \mathrm{g} \mathrm{mL} \mathrm{m}^{-1}$. Hence, DPHC containing extracts of I. okamurae could hold promising anti-inflammatory potential and could be marketed as a cosmeceutical ingredient.

\section{ACKNOWLEDGEMENTS}

This research was supported by Basic Science Research Program through the National Research Foundation of Korea (NRF) funded by the Ministry of Education (NRF2016R1D1A1A02937492).

\section{REFERENCES}

Akdis, M., Aab, A., Altunbulakli, C., Azkur, K., Costa, R. A., Crameri, R., Duan, S., Eiwegger, T., Eljaszewicz, A., Ferstl, R., Frei, R., Garbani, M., Globinska, A., Hess, L., Huitema, C., Kubo, T., Komlosi, Z., Konieczna, P., Kovacs, N., Kucuksezer, U. C., Meyer, N., Morita, H., Olzhausen, J., O’Mahony, L., Pezer, M., Prati, M., Rebane, A., Rhyner, C., Rinaldi, A., Sokolowska, M., Stanic, B., Sugita, K., Treis, A., van de Veen, W., Wanke, K., Wawrzyniak, M., Wawrzyniak, P., Wirz, O. F., Zakzuk, J. S. \& Akdis, C. A. 2016. Interleukins (from IL-1 to IL-38), interferons, transforming growth factor $\beta$, and TNF- $\alpha$ : receptors, functions, and roles in diseases. J. Allergy Clin. Immunol. 138:984-1010.

Barker, J. N. W. N., Griffiths, C. E. M., Nickoloff, B. J., Mitra, R. S., Dixit, V. M. \& Nickoloff, B. J. 1991. Keratinocytes as initiators of inflammation. Lancet 337:211-214.

Bekki, K., Ito, T., Yoshida, Y., He, C., Arashidani, K., He, M., Sun, G., Zeng, Y., Sone, H., Kunugita, N. \& Ichinose, T. 2016. $\mathrm{PM}_{25}$ collected in China causes inflammatory and oxidative stress responses in macrophages through the multiple pathways. Environ. Toxicol. Pharmacol. 45:362369.

Choi, J. C., Lee, M., Chun, Y., Kim, J. \& Oh, S. 2001. Chemical composition and source signature of spring aerosol in Seoul, Korea. J. Geophys. Res. Atmos. 106:18067-18074.

Doelman, C. J. A., Leurs, R., Oosterom, W. C. \& Bast, A. 1990. Mineral dust exposure and free radical-mediated lung damage. Exp. Lung Res. 16:41-55.

Dooms-Goossens, A. E., Debusschere, K. M., Gevers, D. M., Dupré, K. M., Degreef, H. J., Loncke, J. P. \& Snauwaert, J. E. 1986. Contact dermatitis caused by airborne agents. J. Am. Acad. Dermatol. 15:1-10.

Eom, S.-H., Moon, S.-Y., Lee, D.-S., Kim, H.-J., Park, K., Lee, E.-W., Kim, T. H., Chung, Y.-H., Lee, M.-S. \& Kim, Y.-M. 2015. In vitro antiviral activity of dieckol and phlorofucofuroeckol-A isolated from edible brown alga Eisenia bicyclis against murine norovirus. Algae 30:241-246.

Fernando, I. P. S., Kim, M., Son, K. -T., Jeong, Y. \& Jeon, Y. -J. $2016 a$. Antioxidant activity of marine algal polyphenolic compounds: a mechanistic approach. J. Med. Food 19:615-628.

Fernando, I. P. S., Nah, J. W. \& Jeon, Y. J. 2016b. Potential antiinflammatory natural products from marine algae. Environ. Toxicol. Pharmacol. 48:22-30.

Fernando, I. P. S., Sanjeewa, K. K. A., Samarakoon, K. W., Lee, W. W., Kim, H. -S., Kang, N., Ranasinghe, P., Lee, H. -S. \& Jeon, Y. -J. 2017a. A fucoidan fraction purified from Chnoospora minima: a potential inhibitor of LPS-in- 
duced inflammatory responses. Int. J. Biol. Macromol. 104:1185-1193.

Fernando, I. P. S., Sanjeewa, K. K. A., Samarakoon, K. W., Lee, W. W., Kim, H. -S., Kim, E. -A., Gunasekara, U. K. D. S. S., Abeytunga, D. T. U., Nanayakkara, C., de Silva, E. D., Lee, H. -S. \& Jeon, Y. -J. 2017b. FTIR characterization and antioxidant activity of water soluble crude polysaccharides of Sri Lankan marine algae. Algae 32:75-86.

Fujiwara, N. \& Kobayashi, K. 2005. Macrophages in Inflammation. Curr. Drug Targets Inflamm. Allergy 4:281-286.

Han, S. -C., Kang, N. -J., Kang, G. -J., Koh, Y. -S., Hyun, J. -W., Lee, N. -H., Kang, H. -K. \& Yoo, E. -S. 2014. 71: Anti-inflammatory effect of diphlorethohydroxycarmalol (DPHC) isolated from lshige okamuarae in vitro and in vivo. Cytokine 70:44-45.

Heo, S. -J., Hwang, J. -Y., Choi, J. -I., Han, J. -S., Kim, H. -J. \& Jeon, Y. -J. 2009. Diphlorethohydroxycarmalol isolated from Ishige okamurae, a brown algae, a potent $\alpha$-glucosidase and $\alpha$-amylase inhibitor, alleviates postprandial hyperglycemia in diabetic mice. Eur. J. Pharmacol. 615:252-256.

Heo, S. -J., Kim, J. -P., Jung, W. -K., Lee, N. -H., Kang, H. -S., Jun, E. -M., Park, S. -H., Kang, S. -M., Lee, Y. -J., Park, P. -J. \& Jeon, Y. -J. 2008. Identification of chemical structure and free radical scavenging activity of diphlorethohydroxycarmalol isolated from a brown alga, Ishige okamurae. J. Microbiol. Biotechnol. 18:676-681.

Heo, S. -J., Ko, S. -C., Kang, S. -M., Cha, S. -H., Lee, S. -H., Kang, D. -H., Jung, W. -K., Affan, A., Oh, C. \& Jeon, Y. -J. 2010. Inhibitory effect of diphlorethohydroxycarmalol on melanogenesis and its protective effect against UV-B radiation-induced cell damage. Food Chem. Toxicol. 48:1355-1361.

Jalava, P. I., Salonen, R. O., Pennanen, A. S., Sillanpää, M., Hälinen, A. I., Happo, M. S., Hillamo, R., Brunekreef, B., Katsouyanni, K., Sunyer, J. \& Hirvonen, M. -R. 2007. Heterogeneities in inflammatory and cytotoxic responses of RAW 264.7 macrophage cell line to urban air coarse, fine, and ultrafine particles from six European sampling campaigns. Inhal. Toxicol. 19:213-225.

Kandaswami, C., Morin, G. \& Sirois, P. 1988. Lipid peroxidation in rat alveolar macrophages exposed to chrysotile fibres. Toxicol. In Vitro 2:117-120.

Kim, B. -G., Han, J. -S. \& Park, S. -U. 2001. Transport of $\mathrm{SO}_{2}$ and aerosol over the Yellow sea. Atmos. Environ. 35:727737.

Kim, H. -H., Kim, H. -S., Ko, J. -Y., Kim, C. -Y., Lee, J. -H. \& Jeon, Y. -J. 2016. A single-step isolation of useful antioxidant compounds from Ishige okamurae by using centrifugal partition chromatography. Fish. Aquat. Sci. 19:22.
Kim, S. -Y., Kim, E. -A., Kang, M. -C., Lee, J. -H., Yang, H. -W., Lee, J. -S., Lim, T. I. \& Jeon, Y. -J. 2014. Polyphenol-rich fraction from Ecklonia cava (a brown alga) processing by-product reduces LPS-induced inflammation in vitro and in vivo in a zebrafish model. Algae 29:165-174.

Lee, J. -H., Ko, J. -Y., Oh, J. -Y., Kim, E. -A., Kim, C. -Y. \& Jeon, Y. -J. 2015a. Evaluation of phlorofucofuroeckol-A isolated from Ecklonia cava (Phaeophyta) on anti-lipid peroxidation in vitro and in vivo. Algae 30:313-323.

Lee, S. -H., Kang, S. -M., Sok, C. H., Hong, J. T., Oh, J. -Y. \& Jeon, Y. -J. 2015b. Cellular activities and docking studies of eckol isolated from Ecklonia cava (Laminariales, Phaeophyceae) as potential tyrosinase inhibitor. Algae 30:163-170.

Lee, Y. G., Ho, C. -H., Kim, J. -H. \& Kim, J. 2015c. Quiescence of Asian dust events in South Korea and Japan during 2012 spring: dust outbreaks and transports. Atmos. Environ. 114:92-101.

Li, Q. \& Verma, I. M. 2002. NF- $\kappa$ B regulation in the immune system. Nat. Rev. Immunol. 2:725-734.

Monn, C. \& Becker, S. 1999. Cytotoxicity and induction of proinflammatory cytokines from human monocytes exposed to fine (PM2.5) and coarse particles (PM10-2.5) in outdoor and indoor air. Toxicol. Appl. Pharmacol. 155:245-252.

Moreira dos Santos, C. Y., de Almeida Azevedo, D. \& de Aquino Neto, F. R. 2004. Atmospheric distribution of organic compounds from urban areas near a coal-fired power station. Atmos. Environ. 38:1247-1257.

Nel, A. E., Diaz-Sanchez, D., Ng, D., Hiura, T. \& Saxon, A. 1998. Enhancement of allergic inflammation by the interaction between diesel exhaust particles and the immune system. J. Allergy Clin. Immunol. 102:539-554.

Oh, J. -Y., Fernando, I. P. S. \& Jeon, Y. -J. 2016. Potential applications of radioprotective phytochemicals from marine algae. Algae 31:403-414.

Pozzi, R., De Berardis, B., Paoletti, L. \& Guastadisegni, C. 2003. Inflammatory mediators induced by coarse (PM2.5-10) and fine (PM2.5) urban air particles in RAW 264.7 cells. Toxicology 183:243-254.

Sanjeewa, K. K. A., Fernando, I. P. S., Kim, E. -A., Ahn, G., Jee, Y. \& Jeon, Y. -J. 2016a. Anti-inflammatory activity of a sulfated polysaccharide isolated from an enzymatic digest of brown seaweed Sargassum horneri in RAW 264.7 cells. Nutr. Res. Pract. 11:3-10.

Sanjeewa, K. K. A., Fernando, I. P. S., Samarakoon, K. W., Lakmal, H. H. C., Kim, E.-A., Kwon, O.-N., Dilshara, M. G., Lee, J.-B. \& Jeon, Y.-J. 2016b. Anti-inflammatory and anti-cancer activities of sterol rich fraction of cultured marine microalga Nannochloropsis oculata. Algae 
31:277-287.

Schuetz, L. 1989. Atmospheric mineral dust: properties and source markers. In Leinen, M. \& Sarnthein, M. (Eds.) Paleoclimatology and Paleometeorology: Modern and Past Patterns of Global Atmospheric Transport. Springer Netherlands, Dordrecht, pp. 359-383.

Schwartz, J., Dockery, D. W. \& Neas, L. M. 1996. Is daily mortality associated specifically with fine particles? J. Air Waste Manag. Assoc. 46:927-939.

Vallyathan, V., Shi, X., Dalal, N. S., Irr, W. \& Castranova, V. 1988. Generation of free radicals from freshly fractured silica dust: potential role in acute silica-induced lung injury. Am. Rev. Respir. Dis. 138:1213-1219.
Wijesinghe, W. A. J. P., Kang, M. -C., Lee, W. -W., Lee, H. -S., Kamada, T., Vairappan, C. S. \& Jeon, Y. -J. 2014. $5 \beta$-Hydroxypalisadin B isolated from red alga Laurencia snackeyi attenuates inflammatory response in lipopolysaccharide-stimulated RAW 264.7 macrophages. Algae 29:333-341.

Williams, I. R. \& Kupper, T. S. 1996. Immunity at the surface: homeostatic mechanisms of the skin immune system. Life Sci. 58:1485-1507.

Zbytek, B., Mysliwski, A., Slominski, A., Wortsman, J., Wei, E. T. \& Mysliwska, J. 2002. Corticotropin-releasing hormone affects cytokine production in human HaCaT keratinocytes. Life Sci. 70:1013-1021. 